\title{
Quantum Phase Transitions and Collective Modes in $d$-wave Superconductors
}

\author{
Matthias Vojta ${ }^{1}$ and Subir Sachdev ${ }^{2,3}$ \\ 1 Theoretische Physik III, Elektronische Korrelationen und Magnetismus, Institut \\ für Physik, Universität Augsburg, D-86135 Augsburg, Germany \\ 2 Department of Physics, Yale University, P.O. Box 208120, New Haven, CT \\ 06520-8120, USA \\ 3 Department of Physics, Harvard University, Cambridge, MA 02138, USA
}

\begin{abstract}
Fluctuations near second-order quantum phase transitions in $d$-wave superconductors can cause strong damping of fermionic excitations, as observed in photoemission experiments. The damping of the gapless nodal quasiparticles can arise naturally in the quantum-critical region of a transition with an additional spin-singlet, zero momentum order parameter; we argue that the transition to a $d_{x^{2}-y^{2}}+i d_{x y}$ pairing state is the most likely possibility in this category. On the other hand, the gapped antinodal quasiparticles can be strongly damped by the coupling to antiferromagnetic spin fluctuations arising from the proximity to a Neel-ordered state. We review some aspects of the low-energy field theories for both transitions and the corresponding quantum-critical behavior. In addition, we discuss the spectral properties of the collective modes associated with the proximity to a superconductor with $d_{x^{2}-y^{2}}+i d_{x y}$ symmetry, and implications for experiments.
\end{abstract}

\section{Introduction}

The unusual properties of quasiparticle excitations in the cuprate high temperature superconductors, and their possible connection with zerotemperature phase transitions in these systems, have been the subject of intense debate over the past few years. Angle-resolved photoemission (ARPES) experiments fail to find well-defined quasiparticles above the superconducting transition temperature $T_{c}$, and also below $T_{c}$ a simple BCS-like description does not seem to apply. In this context one has to distinguish the quasiparticles excitations in $(1,1)$ direction in momentum space (gapless nodal quasiparticles of the $d$-wave superconductor) from the ones in $(1,0)$ or $(0,1)$ direction (gapped antinodal quasiparticles), see Fig. 17a. ARPES results [1] indicate that the nodal quasiparticles have very short lifetimes in the superconducting state, with their spectral functions having linewidths of order $k_{B} T$, and there is little change [2,3 in this behavior when tuning $T$ through $T_{c}$. The antinodal quasiparticles are broad and ill-defined above $T_{c}$ 邨, but appear to narrow significantly below $T_{c}$ [2, ह], forming long-lived states with an energy gap of $30-40 \mathrm{meV}$.

To explain these unusual properties, two main paradigms have been suggested: either (i) the systems reflect the properties of a fundamentally new 
a)

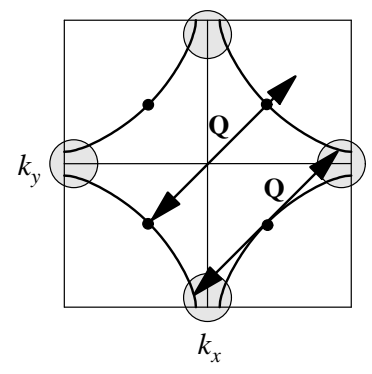

b) $T$

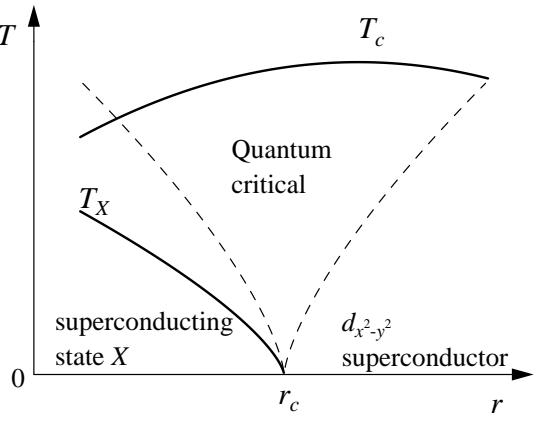

Fig. 1. a) Location of the nodal (solid dots) and antinodal (shaded areas) quasiparticles in the Brillouin zone. The arrows indicate possible scattering of fermions by order parameter fluctuations with wavevector $\mathbf{Q}=(\pi, \pi)$. b) Finite-temperature phase diagram [6] of the $d$-wave superconductor close to a quantum critical point. Superconductivity is present for $T<T_{c}$. The long-range order associated with the state $X$ vanishes for $T>T_{X}$, but fluctuations of this order provide anomalous damping of the nodal quasiparticles in the quantum-critical region. The tuning parameter $r$ is some coupling constant in the Hamiltonian - it is possible, although not necessary, that increasing $r$ corresponds to increasing doping concentration, $\delta$.

state of matter, possibly only contiguous to the superconducting state [7, \&] or (ii) the physics of the stable ground states can be understood in the framework of BCS-like pairing, and many of the unusual finite $T$ experimental properties arise from nearby quantum critical points which mask the behavior of the stable phases of the system 996 . Here, we shall follow the second line of thought, i.e., we assume that the stable phases have no "exotic" properties or excitations, and can (in principle) be described by an appropriate electron Hartree-Fock/RPA/BCS theory with perturbative corrections.

The purpose of this paper is to discuss quantum phase transitions in the high- $T_{c}$ compounds, and their possible connection to the anomalous quasiparticle properties as seen in ARPES experiments [1 5]. For reasons explained below, we will concentrate on transitions related to the onset (a) of addi-

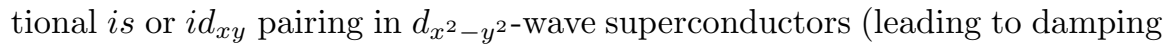
of nodal quasiparticles) and (b) of antiferromagnetic order (leading to damping of antinodal quasiparticles). Furthermore, we will discuss in more detail some properties of a $d_{x^{2}-y^{2}}+i d_{x y}$ superconductor close to the quantum critical point (a), and derive its collective excitation spectrum.

The first quantum phase transition studied in the context of cuprate superconductors was the destruction of Néel order by doping. This transition has been proposed [9, 15] to belong to the same universality class as the order-disorder transition in insulating antiferromagnets driven by quantum fluctuations 16 . This implies a dynamic critical exponent $z=1$, and a stable $S=1$ "resonant" spin excitation near the antiferromagnetic wavevector in the paramagnetic state [9, 17], both being consistent with numerous neutron 
scattering and NMR studies. Assuming that the mobile charge carriers have a $d$-wave superconducting ground state on both sides of the transition, the arguments for the common universality of the magnetic quantum critical point in insulating and doped antiferromagnets can be sharpened. The order parameter is a 3-component real field, $N_{\alpha}$, which measures the amplitude of the local antiferromagnetic order. The onset of long-range antiferromagnetism is described by the condensation of $N_{\alpha}$, and the theory for the quantum critical point will depend upon whether the $N_{\alpha}$ couple efficiently to the low-energy fermionic excitations of the $d$-wave superconductor (the $S=1 / 2$ Bogoliubov quasiparticles). Momentum conservation now plays a key role: the dominant fluctuations of $N_{\alpha}$ occur at the ordering wavevector $\mathbf{Q}$, and the fermions will be scattered by this the wavevector. If $\mathbf{Q}$ does not equal the separation between two nodal points of the $d$-wave superconductor [located at momenta $( \pm K, \pm K$ ) with $K=0.39 \pi$ at optimal doping, see Fig [1]a], then the fermion scattering does not lead to any disruptive low energy damping of the $N_{\alpha}$. In such a situation, there is no fundamental difference between the low-energy magnetic fluctuations in a superconductor and an insulating paramagnet, and the same theory for the antiferromagnetic quantum transition applies. Of course, these arguments also hold if the charge sector is fully gapped on both sides of the transition, i.e., if the ground state around the magnetic quantum critical point is insulating.

The magnetic quantum phase transition alone is not likely to explain the plethora of unusual finite- $T$ properties of the cuprates. Instead, it appears that a variety of ordered phases compete in these two-dimensional doped Mott insulators, and numerous additional transitions are possible and have been discussed in recent years: the onset of site/bond charge and/or spin density wave order ("stripes") [10], additional superconducting order like $d+i s$ or $d+i d$ pairing [18], excitonic (or nematic) order [19], staggered flux (or $d$-density wave) order [11, 20, and "exotic" transitions based on the concept of spin-charge separation [7,21]. Except for the latter, all these transitions can be characterized by an order parameter which is assumed to carry a net momentum $\mathbf{Q}$ corresponding to the ordering wavevector. Whereas superconducting and excitonic order have $\mathbf{Q}=0$, stripe order is characterized by a finite (possibly incommensurate) wavevector $\mathbf{Q}$, and staggered flux order corresponds to $\mathbf{Q}=(\pi, \pi)$ (similar to antiferromagnetic Neel order).

We now turn to the influence of these possible quantum phase transitions on the properties of the fermionic quasiparticles, and we will first concentrate on the nodal, i.e., the low-energy, fermions. In the vicinity of a quantum transition the fermions will be strongly scattered by bosonic order parameter fluctuations. Similar to the discussion above, two general cases have to be distinguished: Either (A) the low-energy fermions are scattered into fermion states with higher energy, or (B) scattering occurs between lowenergy fermions, i.e., the wavevector $\mathbf{Q}$ must equal the momentum space separation between two nodal points (nesting condition). In case (A) fermion 
scattering events can be treated as virtual processes, and the critical theory of the quantum phase transition is not fundamentally modified by the presence of the fermions. A perturbative expansion for the fermionic self-energy is well behaved, and the fermion damping will vanish with a super-linear power of temperature $(T)$ as $T \rightarrow 0$ [6]. In contrast, in case (B) the fermions become part of the critical theory, a perturbative expansion in the coupling is infrared singular, and a correct treatment requires a coupled critical theory of bosons and fermions. The most efficient quasiparticle scattering is provided by a linear, non-derivative coupling between fermion bilinears and order parameter bosons [6]. If such a coupling is relevant in the renormalization group sense, then one expects quantum-critical damping of the fermions, i.e., the damping rate will vanish linearly with $T$.

In Sec 2 we review theories for the quantum-critical damping of nodal fermions, belonging to case (B). We shall find that a transition to a state with $d_{x^{2}-y^{2}}+i d_{x y}$ pairing is the most likely candidate in this category. The damping of antinodal fermions is subject of Sec 3; we will restrict the discussion to effects arising from the proximity to a magnetic phase transition. Finally, in Sec 1 we return to a $d_{x^{2}-y^{2}}+i d_{x y}$ superconductor and discuss collective modes arising from fluctuations of the additional order parameter component close to the critical point.

\section{Damping of Nodal Quasiparticles}

Motivated by the ARPES experiments on $\mathrm{Bi}_{2} \mathrm{Sr}_{2} \mathrm{CaCu}_{2} \mathrm{O}_{8+\delta}$ [1] which indicate a nodal fermion scattering rate being proportional to $T$, we are interested quantum-critical damping of the nodal fermions [case (B) above]. It arises from fluctuations near a quantum critical point between the $d_{x^{2}-y^{2}}$ superconductor and some other superconducting state $X$ (see Fig $1 \mathrm{~b}$ ). The corresponding critical theories allow for a well-controlled treatment using renormalization group ( $R G$ ) techniques. The reason is the restricted phase space for low-energy excitations in a $d$-wave superconductor; in a metallic system [where case (B) is the generic situation] the situation is much more complicated due to the presence of low-energy particle-hole excitations at arbitrary momenta.

As discussed above, quantum-critical scattering requires that the momentum $\mathbf{Q}$ carried by the order parameter connects two nodal points. Three natural possibilities can satisfy this nesting condition: $\mathbf{Q}=0, \mathbf{Q}=(2 K, 2 K)$, and $\mathbf{Q}=(2 K, 0),(0,2 K)$. Importantly, $K$ depends on microscopic parameters, therefore the latter two require fine-tuning, unless there is a mode-locking between the values of $\mathbf{Q}$ and $K$. Candidates for $\mathbf{Q}=(2 K, 2 K)$ are Neel order and staggered-flux order 11, 20, both have $\mathbf{Q}=(\pi, \pi)$, and nesting is apparently not satisfied near optimal doping. In addition, staggered-flux order leads to a derivative coupling between nodal fermions and the order parameter which can be shown to be irrelevant 22]. Transitions involving the onset 
of spin 21] or site/bond charge density waves [6] (stripes) can possibly satisfy $\mathbf{Q}=(2 K, 0),(0,2 K)$, however, this restriction on $\mathbf{Q}$ is not realized by the $\mathbf{Q}$ values observed so far.

We are left with $\mathbf{Q}=0$ being the only possibility which can naturally satisfy the momentum conservation constraints for a range of parameter values. Assuming that the order parameter is a $\mathbf{Q}=0$ spin-singlet fermion bilinear (spin triplet condensation at $\mathbf{Q}=0$ would imply ferromagnetic correlations which are unlikely to be present), group theoretic arguments [22] permit a complete classification of such order parameters. The order parameter for $X$ must be built out of the following correlators $\left(c_{\mathbf{q} a}\right.$ annihilates an electron with momentum $\mathbf{q}$ and $\operatorname{spin} a=\uparrow, \downarrow)$

$$
\left\langle c_{\mathbf{q} a}^{\dagger} c_{\mathbf{q} a}\right\rangle=A_{\mathbf{q}} \quad \text { and } \quad\left\langle c_{\mathbf{q} \uparrow} c_{-\mathbf{q} \downarrow}\right\rangle=\left[\Delta_{0}\left(\cos q_{x}-\cos q_{y}\right)+B_{\mathbf{q}}\right] e^{i \varphi},
$$

where $\Delta_{0}$ is the background $d_{x^{2}-y^{2}}$ pairing which is assumed to be non-zero on both sides of the transition, $\varphi$ is the overall phase of the superconducting order, and $A_{\mathbf{q}}$ and $B_{\mathbf{q}}$ contain the possible order parameters for the state $X$ corresponding to condensation in the particle-hole (or excitonic) channel or additional particle-particle pairing, respectively. The functions $A_{\mathbf{q}}$ and $B_{\mathbf{q}}$ can now be expanded in terms of the basis functions of the irreducible representation of the tetragonal point group $C_{4 v}$, and this leads to seven distinct order parameters for the state $X$ [22]. The corresponding field theories have been analyzed recently [6, 22 by means of RG techniques, and the results are simple and remarkable. Only for two cases, namely the transitions between a $d_{x^{2}-y^{2}}$-wave superconductor and a state with either $d_{x^{2}-y^{2}}+i s$ or $d_{x^{2}-y^{2}}+i d_{x y}$ pairing, there exists a fixed point describing a second-order quantum phase transition where the fermions are part of the critical theory. For all other cases, we either found runaway flows of the couplings, with no non-trivial fixed points, or a fixed point where the fermions are decoupled from the critical degrees of freedom.

We now describe some aspects of the critical theory for the $d_{x^{2}-y^{2}}+i s$ and $d_{x^{2}-y^{2}}+i d_{x y}$ cases. In the quantum critical region (Fig $1 \mathrm{l} \mathrm{b}$ ) the single fermion Green's functions being measured in photoemission experiments will obey the scaling form

$$
G_{f}(k, \omega)=\frac{\mathcal{A}_{f}}{T^{1-\eta_{f}}} \Phi_{f}\left(\frac{\omega}{T}, \frac{k}{T}\right),
$$

where we have set $\hbar, k_{B}$ and all velocities to unity. The scale factor $\mathcal{A}_{f}$ is nonuniversal, while the anomalous exponent $\eta_{f}$ and the complex-valued function $\Phi_{f}$ are universal. The nodal fermions and the order parameter fluctuations are strongly coupled, and the anomalous dimension of the fermion field, $\eta_{f} / 2$, leads to a large $\omega$ tail in its energy distribution curve (EDC) [6, 23, 24], which is actually consistent with ARPES measurement. The scaling function $\Phi_{f}$ has been studied in Ref [6]; for $\omega, k \gg T$, (2) reduces to [6, 21]

$$
G_{f}(k, \omega)=\mathcal{A} C_{f} \frac{\omega+k_{x} \tau^{z}+k_{y} \tau^{x}}{\left[k^{2}-(\omega+i 0)^{2}\right]^{1-\eta_{f} / 2}}
$$



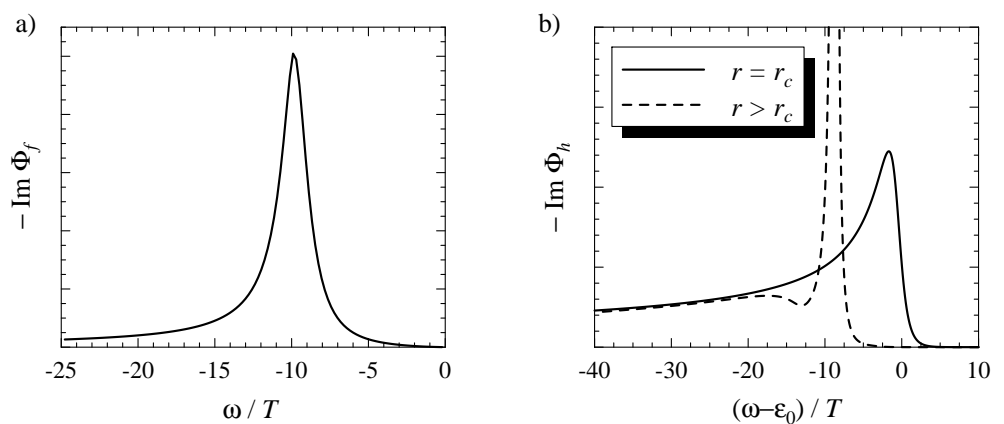

Fig. 2. Scaling functions describing the fermion damping. a): Fermion spectral density near a nodal point in the quantum critical region described in Sec 2 , in the regime $\omega, k \gg T$ (here $k / T=10$ ) [6]. Right: Antinodal fermion spectral density near the antiferromagnetic ordering transition (Sec 3), both in the critical region $r=r_{c}$ and in the regime with gapped fluctuations $r>r_{c}(\Delta / T=5)$ 27. In both cases the asymmetry of the lineshapes and the tail at higher binding energies is clearly visible.

where $C_{f}$ is a universal number. Note that the imaginary part of this is non-zero only for $\omega>k$, and it decays as $\omega^{-1+\eta_{f}}$ for large $\omega$. A result for the scaling function in this regime is shown in Fig 2 2 a. In the opposite limit, $\omega, k \ll T$, (2) has a very different form, and the $k$ and $\omega$ dependencies are smooth. For further discussion we refer the reader to Ref [6].

Notably, the theories for both cases, $d_{x^{2}-y^{2}}+i s$ and $d_{x^{2}-y^{2}}+i d_{x y}$ pairing, lead to identical damping behavior for the nodal fermions. However, they can be distinguished from their effect on the antinodal quasiparticles: $i s$ fluctuations will couple to all fermions, whereas the $i d_{x y}$ order parameter has nodes in the $(1,0),(0,1)$ directions, therefore leaving the antinodal fermions essentially unaffected. With reference to the ARPES result about sharp antinodal quasiparticles for $T \ll T_{c}$, we can uniquely propose the $d_{x^{2}-y^{2}}+i d_{x y}$ transition as explanation for the nodal fermion damping below $T_{c}$.

\section{Damping of Antinodal Quasiparticles}

In this section, we turn to the anomalous behavior of the antinodal fermions. ARPES experiments show a broad EDC above $T_{c}$, whereas below $T_{c}$ a quasiparticle-like peak appears separated from an incoherent tail, and the spectrum has been described as "peak-dip-hump" structure [2, 4 , 0 ].

For $T \ll T_{c}$ the antinodal quasiparticles live at a finite energy (given by the maximum value of the superconducting gap), and possible causes for damping are less restricted than in the case of the nodal fermions. We will concentrate here on fermion scattering by antiferromagnetic fluctuations 10,25]; other proposals include one-dimensional [24] or two-dimensional [7] electron 
fractionalization (induced by proximity to spin liquid states) and the coupling to superconducting phase and vortex fluctuations [26], these will not be discussed here.

It can be seen from Fig 1 1 a that magnetic fluctuations with wavevector $\mathbf{Q}=(\pi, \pi)$ can provide an efficient coupling between antinodal quasiparticles. We will examine their spectral function (or EDC) in the vicinity of a quantum critical point between a $d$-wave superconductor and a state with co-existing superconducting and antiferromagnetic order. Appealing to the proximity of a magnetic quantum critical point allows us to make controlled statements in a regime with strong coupling between the fermionic quasiparticles and the antiferromagnetic fluctuations [27].

As argued in the introduction, the critical theory of the antiferromagnetic transition itself is not influenced by the presence of fermions, because the order parameter fluctuations, $N_{\alpha}$, with momentum $\mathbf{Q}=(\pi, \pi)$ do not scatter between low-energy nodal fermions of the superconductor. Therefore, in contrast to the theories of Sec 2, we can work here with the "bare" bosonic theory for the ordering transition, which will now be coupled to a single antinodal fermion ("hole") [27]. Further simplifications arise from the assumption that the fermion momentum corresponds to an extremum or van-Hove point of the band structure. Then, the dispersion has no linear momentum dependence, and RG analysis shows that quadratic and higher terms in the dispersion are irrelevant, therefor the hole may be viewed as dispersionless (immobile). Also, the coupling between the charge density of the fermion and the bulk theory is irrelevant under RG. The resulting theory has the structure of a Bose-Kondo-like model [15,28] of a single quantum spin coupled locally to the bosonic fluctuations of a nearly critical antiferromagnet. (A similar boundary field theory has been studied [28] in the context of $\mathrm{Zn} / \mathrm{Li}$ impurities in cuprate superconductors.)

We now turn to the hole spectrum as measured in photoemission. At the $T=0$ quantum critical point at $r=r_{c}$, the $N_{\alpha}$ are gapless critical excitations, and a naive perturbation expansion of the hole self-energy is infrared singular. Our recent RG analysis [27] of the scale-invariant quantumfield theory permits a resummation of the perturbative expansion. In the $r \geq r_{c}, T \geq 0$ vicinity of the critical point, the hole Green's function obeys the scaling form

$$
G_{h}(\omega)=\frac{\mathcal{A}_{h}}{T^{1-\eta_{h}}} \Phi_{h}\left(\frac{\omega-\epsilon_{0}}{T}, \frac{\Delta}{T}\right)
$$

where $\Delta$ is the spin gap of the host antiferromagnet, $\mathcal{A}_{h}$ is a non-universal amplitude and $\epsilon_{0}$ denotes the bare fermion energy at the antinodal point. The anomalous exponent $\eta_{h}$ and the scaling function $\Phi_{h}$ are again universal. In particular, at the critical point $\left(r=r_{c}, T=0\right)$ there is no quasiparticle pole, but a power-law singularity described by $G_{h}=-\mathcal{A}_{h}\left(\epsilon_{0}-\omega\right)^{-1+\eta_{h}}$ ( $\operatorname{spin}$ orthogonality catastrope). Numerical results for $\Phi_{h}$ obtained with a large $N$ method [28] are displayed in Fig 2b. The $\mathbf{k}$ dependence of $G_{h}$ arises only 
from the irrelevant band curvature terms, and their main effect is to replace $\epsilon_{0}$ by the actual hole dispersion near the van-Hove point. We expect that the $r=r_{c}$ spectrum in Fig $2 \mathrm{~b}$ applies to photoemission at the antinodal points above $T_{c}$. Below $T_{c}$, the measured antinodal spectrum [2] is similar to the $r>r_{c}$ spectrum in Fig 2 $2 \mathrm{~b}$ : this is accounted for in our approach by the reasonable assumption that the onset of superconductivity induces the spingap-like correlations and so increases the value of the effective $r$ controlling the magnetic fluctuations. The high frequency tail of the EDC both above and below $T_{c}$ should decay as $1 / \omega^{1-\eta_{h}}$.

\section{Collective Modes Associated with $d_{x^{2}-y^{2}}+i d_{x y}$ Pairing}

In this section, we focus on the $d_{x^{2}-y^{2}}+i d_{x y}$ superconductor which emerged from our discussion of nodal quasiparticle damping in Sec 2. The aim is to identify the low-energy collective modes which should exist near the quantum phase transition to a state with pure $d_{x^{2}-y^{2}}$ pairing, and to discuss experimental signatures of these modes. Such collective modes have also been considered recently by Balatsky et al. [29], but our results below differ significantly from theirs.

To proceed, we have to derive an effective action for the fluctuations of the $d_{x y}$ order parameter. We will demonstrate this explicitely on the basis of a $\operatorname{Sp}(N)$ mean-field theory [6] for a $t$ - $J$ model with additional diagonal exchange interaction, $J_{2}$, although the results discussed below are far more general. We start in a regime where the ground state is a pure $d_{x^{2}-y^{2} \text {-wave }}$ superconductor. It is described by a saddle point of the large- $N$ theory, and superconductivity is encoded in non-zero pairing amplitudes on the links of the square lattice. Now we consider a perturbation which introduces pairing along the diagonals, with an amplitude $Q_{1,1}=-Q_{1,-1} \equiv Q_{x y}$, where $Q_{x y}$ is a space and time dependent complex number. We factor out the overall phase of the superconducting order, $\varphi$, as in (17), and so the phase of $Q_{x y}$ measures the relative phase between the $d_{x y}$ and $d_{x^{2}-y^{2}}$ orders. The fluctuations of $\varphi$ couple to overall charge fluctuations and these occur at a high plasma frequency - we can therefore neglect $\varphi$ in our considerations. These arguments encapsulate the physics discussed by Balatsky et al. in terms of numbers of Cooper pairs with $d_{x y}$ and $d_{x^{2}-y^{2}}$ pairing.

After integrating out the fermions in the $d$-wave saddle point, the large- $N$ theory gives an effective action for $Q_{x y}$ of the following form:

$$
\begin{aligned}
S_{Q}=\sum_{\mathbf{k}, \omega_{n}}\{ & I\left(\mathbf{k}, \omega_{n}\right) Q_{x y}\left(\mathbf{k}, \omega_{n}\right) Q_{x y}^{*}\left(\mathbf{k}, \omega_{n}\right) \\
& \left.+\frac{1}{2}\left[J\left(\mathbf{k}, \omega_{n}\right) Q_{x y}\left(\mathbf{k}, \omega_{n}\right) Q_{x y}\left(-\mathbf{k},-\omega_{n}\right)+H . c\right]\right\},
\end{aligned}
$$


a)

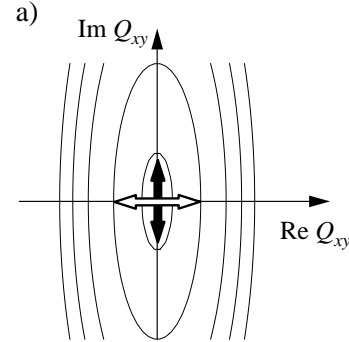

$B=0, r>r_{c}$ b)

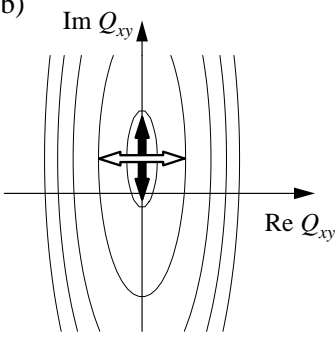

$B=0, r<r_{c}$

or $B>0, r>r_{c}$ c)

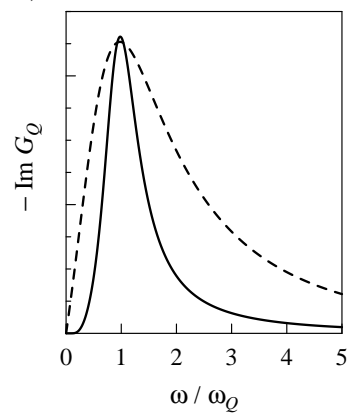

Fig. 3. a) Schematic contour plot of the Landau-Ginzburg free energy for the fluctuations of real and imaginary part of the $d_{x y}$ order parameter component, $Q_{x y}=\psi+i \phi$, for zero magnetic field $B$ and $r>r_{c}$, i.e., in the region where the ground state is a pure $d_{x^{2}-y^{2}}$-wave superconductor. The double arrows denote the normal modes of the $Q_{x y}$ fluctuations. b) Same as a), but in the region with a $d_{x^{2}-y^{2}}+i d_{x y}$ ground state (for $r<r_{c}$ or $B>0, r>r_{c}$ ). c) Spectral density of the zero-momentum $Q_{x y}$ propagator, for $r>r_{c}$, but close to the quantum critical point at $r_{c}$. The peak corresponds to the oscillation of $\phi$ about zero, with a characteristic energy $\omega_{Q}$. Dashed: $T \ll \omega_{Q}$, solid: $T \gg \omega_{Q}$. The excitation corresponding to the oscillation of $\psi$ is strongly overdamped and not visible.

where $I$ and $J$ are complex functions, and have expressions given by simple one-loop fermion diagrams. Explicitely, we find

$$
\begin{aligned}
& I\left(\mathbf{k}, \omega_{n}\right)=\frac{1}{J_{2}}-\sum_{\mathbf{q}, \nu_{n}} \gamma_{x y}^{2}\left(\mathbf{q}-\frac{\mathbf{k}}{2}\right) G\left(\mathbf{q}, \nu_{n}\right) G\left(-\mathbf{q}+\mathbf{k},-\nu_{n}+\omega_{n}\right) \\
& J\left(\mathbf{k}, \omega_{n}\right)=\sum_{\mathbf{q}, \nu_{n}} \gamma_{x y}^{2}\left(\mathbf{q}-\frac{\mathbf{k}}{2}\right) F\left(\mathbf{q}, \nu_{n}\right) F\left(-\mathbf{q}+\mathbf{k},-\nu_{n}+\omega_{n}\right) .
\end{aligned}
$$

Here $G$ and $F$ are the normal and anomalous Green's functions of the $d$-wave superconductor, and $\gamma_{x y}(\mathbf{q})=\cos \left(q_{x}+q_{y}\right)-\cos \left(q_{x}-q_{y}\right)$.

We now study the small momentum and frequency behavior of the $Q$ fluctuations. We perform a small $k$ and $\omega_{n}$ expansion of (5), with the decomposition $Q_{x y}=\psi+i \phi$, and find

$$
\begin{gathered}
S_{Q}=\sum_{\mathbf{k}, \omega_{n}}\left\{\left(C_{1}+C_{1 k}|\mathbf{k}|+C_{1 \omega}\left|\omega_{n}\right|\right) \psi^{2}+\left(C_{2}+C_{2 k}|\mathbf{k}|+C_{2 \omega}\left|\omega_{n}\right|\right) \phi^{2}\right. \\
\left.+C_{3} \omega_{n} \psi \phi+\cdots\right\},
\end{gathered}
$$

where the $C$ 's are some real constants. The most notable terms are the nonanalytic $|\mathbf{k}|$ and $|\omega|$ terms displayed above. The remaining higher order terms are analytic and turn into regular gradients after Fourier transforming to the spacetime representation. The non-analytic terms are a consequence of 
the gapless nodal quasiparticles in the $d_{x^{2}-y^{2}}$ superconductor. Further, an analysis of the infra-red singularities of the nodal fermion fluctuations, using the field-theoretic methods of [22], shows that the nature of the nonanalyticity in (7) is robust, i.e., higher-order corrections do not modify the non-analytic power of the momentum or frequency, or introduce additional non-analyticities. In this sense, these singularities are the fermionic analog of the Goldstone singularities arising from spin waves in the ordered phase of an antiferromagnet. Only at the quantum critical point, at which there is onset of $d_{x y}$ order, are there additional higher-order corrections to (7), which then re-exponentiate to give the anomalous critical exponents as in (33) [22].

We now discuss the behavior of the low-energy action $S_{Q}$ near the quantum critical point involving onset of $d_{x y}$ order. At the transition, fluctuations described by $\phi=\operatorname{Im} Q_{x y}$ condense, and therefore close to the transition we expect $C_{2} \ll C_{1}$ [note $\left.C_{1,2}=(I \pm J)(\mathbf{k}=0, \omega=0)\right]$. This situation is illustrated in Fig $3 \mathrm{a}$, and it is clear that the normal modes for $r>r_{c}$ are oscillations of $\phi$ and $\psi$ about zero, with the $\phi$ mode having a much lower energy. However, the non-analyticities mentioned above imply that the propagator associated with $Q_{x y}$ fluctuations does not have a simple pole structure. We have evaluated the spectral density of this propagator in the large- $N$ theory of Ref [28], and results are shown in Fig 3c. There is a peak at a characteristic energy, $\omega_{Q}$, associated with the $\phi$ oscillations, but it has a rather large width in the zero-temperature limit, $T \ll \omega_{Q}$. In the opposite limit, $T \gg \omega_{Q}$, the peak narrows, but does not become a simple pole. At higher energies, the propagators are strongly damped, and no structure is visible at an energy corresponding to $\psi$ oscillations.

Moving to $r<r_{c}, \operatorname{Im} Q_{x y}$ acquires a finite expectation value, and the resulting Landau-Ginzburg free energy is illustrated in Fig $3 \mathrm{~b}$. Applying a non-zero magnetic field $B$ for $r>r_{c}$ has the same effect (if we ignore vortex physics [30]), i.e., it introduces a term proportional to $B \Delta_{0} \phi$ into the action, and thereby induces a finite $i d_{x y}$ order parameter component. From Fig $3 \mathrm{~b}$ it is clear that the low-energy mode is still given by amplitude fluctuations of $\phi$ whereas the $\psi$ mode is high in energy. Furthermore, the frequency of the $\phi$ oscillations remains $B$ independent for small fields $B$.

These results above differ from those of Ref [29], which considers a "clapping mode" oscillation between the two order parameters. In our notation, as is clear from Fig $3 \mathrm{~b}$, this mode is mainly the $\psi$ mode: we claim that near a transition to the $d_{x^{2}-y^{2}}+i d_{x y}$ state, such a mode will be at high energy and overdamped; our symmetry analysis also shows that it is decoupled from the proper low energy mode, which is the $\phi$ oscillation. Further the field independence of our normal mode frequencies does not agree with [29]. The main reason for these discrepancies seems the absence of the $J\left(\mathbf{k}, \omega_{n}\right)$ term (5,6) in the Landau-Ginzburg free energy of Ref [29], in their notation it would correspond to $\left(\Delta_{0}^{*} \Delta_{1}\right)^{2}+h . c$., which is allowed by symmetry on the square lattice; here $\Delta_{0,1}$ are the $d_{x^{2}-y^{2}}$ and $d_{x y}$ order parameter components. 
Finally, we discuss possible experimental signatures of the $d+i d$ instability. A time-reversal symmetry breaking $d_{x^{2}-y^{2}}+i d_{x y}$ bulk ground state has not been detected so far; so we assume that the system has $r>r_{c}$. A lowenergy $\phi$ oscillation mode should be well defined close to the critical point, it is associated with chiral fluctuations [18]. A comparison with anomalies in transport measurements (see Ref [22]) suggests an energy of order $20 \mathrm{~K}$ for this mode. Therefore we expect a corresponding signal in polarized Raman 31] or neutron scattering [32] where the use of appropriate circular polarizations allows a unique identification of chiral fluctuations.

\section{Conclusion}

In this paper, we have reviewed theories for quantum phase transitions in $d$ wave superconductors which can cause strong damping of low-energy quasiparticles. All these theories are associated with a quantum critical point between a $d$-wave superconductor and some other superconducting state $X$ (Fig 1 $1 \mathrm{~b}$ ). To explain the strong, inelastic scattering of nodal fermions as observed in photoemission well below $T_{c}$, the state $X$ has to be associated with a spin-singlet, zero momentum, fermion bilinear order parameter. We found that only two candidates for $X$ possessed a non-trivial quantum critical point, a $d_{x^{2}-y^{2}}+i s$ or a $d_{x^{2}-y^{2}}+i d_{x y}$ superconductor, where the latter does not affect the antinodal fermions. We have also considered the damping of antinodal fermions arising from the proximity to a Neel ordered state. The fermion scattering from antiferromagnetic fluctuations provides an explanation for the broad lineshapes and the "peak-dip-hump" structure seen in experiment.

Finally we have discussed the collective modes of a $d$-wave superconductor close to a zero temperature $d_{x^{2}-y^{2}}+i d_{x y}$ instability. The only observable lowenergy mode is associated with amplitude fluctuations of the imaginary part of the $d_{x y}$ order parameter component, whereas the suggested "clapping" mode [29] is found to be at high energy and overdamped.

\section{Acknowledgments}

We thank the US NSF (DMR 00-98226) and the DFG (VO 794/1-1 and SFB 484) for support.

\section{References}

1. T. Valla et al., Science 285, 2110 (1999)

2. H. R. Ding et al., Phys. Rev. Lett. 76, 1533 (1996); Z. X. Shen et al., Science 280, 259 (1998); A. Kaminski et al., Phys. Rev. Lett. 84, 1788 (2000); M. R. Norman et al., cond-mat/0012335 (2000) 
3. P. V. Bogdanov et al., Phys. Rev. Lett. 85, 2581 (2000); A. Kaminski et al., ibid 86, 1070 (2001); P. D. Johnson et al., cond-mat/0102260 (2001)

4. Z.-X. Shen et al., Phys. Rev. Lett. 70, 1553 (1993); D. S. Marshall et al., ibid 76, 4841 (1995); H. Ding et al., Nature 382, 51 (1996)

5. J. C. Campuzano et al., Phys. Rev. Lett. 83, 3709 (1999); D. H. Lu et al., cond-mat/0103047 (2001)

6. M. Vojta, Y. Zhang, and S. Sachdev, Phys. Rev. B 62, 6721 (2000)

7. T. Senthil and M. P. A. Fisher, Phys. Rev. B 62, 7850 (2000); cond-mat/9912380

8. J. Zaanen et al., cond-mat/0102103 (2001)

9. S. Sachdev and J. Ye, Phys. Rev. Lett. 69, 2411 (1992); A. V. Chubukov and S. Sachdev, ibid. 71, 169 (1993); A. V. Chubukov, S. Sachdev, and J. Ye, Phys. Rev. B 49, 11919 (1994)

10. C. Castellani, C. DiCastro, and M. Grilli, Phys. Rev. Lett. 75, 4650 (1995); S. Caprara et al., Phys. Rev. B 59, 14980 (1999)

11. D. A. Ivanov, P. A. Lee, and X.-G. Wen, Phys. Rev. Lett. 84, 3958 (2000); S. Chakravarty et al., Phys. Rev. B 63, 094503 (2001)

12. S.-C. Zhang, Science 275, 1089 (1997)

13. R. B. Laughlin, Adv. in Phys. 47, 943 (1998)

14. J. Zaanen, Physica C 317, 217 (1999)

15. S. Sachdev, Science 288, 475 (2000)

16. S. Chakravarty, B. I. Halperin, and D. R. Nelson, Phys. Rev. Lett. 60, 1057 (1988); Phys. Rev. B 39, 2344 (1989)

17. P. W. Anderson, cond-mat/0007185

18. See the review M. Sigrist, Prog. Theor. Phys. 99, 899 (1998)

19. S. A. Kivelson, E. Fradkin, and V. J. Emery, Nature 393, 550 (1998)

20. C. Nayak, Phys. Rev. B 62, 4880 (2000)

21. L. Balents, M. P. A. Fisher, and C. Nayak, Int. J. Mod. Phys. B 12, 1033 (1998)

22. M. Vojta, Y. Zhang, and S. Sachdev, Phys. Rev. Lett. 85, 4940 (2000), Int. J. Mod. Phys. B 14, 3719 (2000)

23. S. Sachdev, Quantum Phase Transitions, Cambridge University Press, Cambridge (1999)

24. D. Orgad et al., cond-mat/0005457 (2000)

25. C. M. Varma, Phys. Rev. Lett. 83, 3538 (1999); L. B. Ioffe and A. J. Millis, Phys. Rev. B 58, 11631 (1998)

26. M. Franz and A. J. Millis, Phys. Rev. B 58, 14572 (1998); H.-J. Kwon and A. T. Dorsey, Phys. Rev. B 59, 6438 (1999); A. Paramekanti et al., Phys. Rev. B 62, $6786(2000)$

27. S. Sachdev, M. Troyer, and M. Vojta, Phys. Rev. Lett. 86, 2617 (2001)

28. M. Vojta, C. Buragohain, and S. Sachdev, Phys. Rev. B 61, 15152 (2000)

29. A. V. Balatsky, P. Kumar, and J. R. Schrieffer, Phys. Rev. Lett. 84, 4445 (2000)

30. M.-R. Li, P. J. Hirschfeld, and P. Wölfle, Phys. Rev. B 63, 054504 (2001)

31. B. S. Shastry and B. I. Shraiman, Phys. Rev. Lett. 65, 1068 (1990); D. V. Khveshchenko and P. B. Wiegmann, ibid 73, 500 (1994); S. Yoon et al., ibid 85, 3297 (2000)

32. S. V. Maleyev, Phys. Rev. Lett. 75, 4682 (1995); V. P. Plakhty et al., ibid 85, 3942 (2000) 